\title{
Good short-term survival of IPS-Empress crowns
}

\author{
How long do IPS-Empress ceramic restorations last?
}

\section{El-Mowafy O, Brochu JF. Longevity and clinical performance of IPS-Empress ceramic restorations - a literature review. J Can Dent Assoc 2002; 68:233-237}

Data sources Medline was the only data source for this review. Study selection Only studies that dealt with inlays, onlays or crowns and that were published in English were included. Studies that lasted less than 2 years were excluded, as were those published in abstract form only.

Data extraction and synthesis The studies were divided into two categories: those that considered inlay and onlay restorations and those that considered crowns. Qualitative assessment was conducted.

Results Nine studies were selected, six of which assessed the performance of inlays and onlays made with the pressable glass matrix IPS-Empress system (Ivoclar Vivadent, Schaan, Liechtenstein; IPSEmpress is the registered trade name of this ceramic restoration) and three of which examined the performance of IPS-Empress crowns. Survival for inlays and onlays ranged from $96 \%$ at 4.5 years to $91 \%$ at 7 years, with most failures being caused by bulk fracture. The survival of crowns ranged from $92 \%$ to $99 \%$ at 3-3.5 years, with failure again being caused primarily by fracture.

Conclusions Use of IPS-Empress crowns is not recommended in the posterior region of the mouth until the results of a sufficient number of long-term clinical trials of premolars and molars are available.

\section{Commentary}

Machinable ceramics offer an alternative to metallic-based restorative materials and composites. They are biocompatible and aesthetically excellent, but are highly technique-sensitive and require extensive tooth preparation.

This literature review only considers the pressable glass matrix IPS-Empress ceramic system. It is important to note that this is not a high-quality systematic review: only Medline was searched and no details of the search strategy are given. It is not clear how many studies were excluded or why, and no attempt was made to contact authors of studies published in abstract only. There is no detail of how many people appraised those studies that were included nor information about the types of study design that were accepted. A wide range of study types including case series and retrospective reports were included, however.

The qualitative analysis is based on six studies for inlays and onlays and three studies for crowns. The results suggest that 5-10\% of inlays and onlays of this material are likely to fracture before 5 years. For these restorations, size and type of tooth do not appear to influence failure. Although marginal ditching was commonly reported, it was not severe enough to warrant replacing the restoration. Up to $8 \%$ of crowns failed within 3 years but, as the majority of crowns were placed on anterior teeth, these results are not applicable to posterior crowns and therefore use of the IPSEmpress for these is not recommended. Almost all failures in the reported studies were due to bulk fracture of the inlay, onlay or crown. Wear of the luting composite, submargination and tooth fracture, which have been reported as causes of failure with other ceramic inlay systems, ${ }^{1}$ do not seem to have been a problem. Although post-operative sensitivity was noted, it was transient and resolved in a maximum of 8 weeks, with only two cases of pulpitis reported and those were in a single study.

Almost all of the nine studies report restorations placed in university clinics, using carefully selected patients who did not have bruxing habits. As such, the results cannot be generalised to all settings and patients. The results suggest that IPS-Empress inlay, onlays and anterior crowns are showing good survival in the short term. In order for clinicians and patients to determine if they are the best restoration for a particular tooth comparative data is required on the alternatives.

Sadly, few reviews of the longevity of inlays or traditional restorations have been reported ${ }^{1-3}$ and it is therefore difficult to determine which restorations are likely to be successful for a given patient. Although randomised controlled trials that compare ceramic inlay systems with other restorative options would be the ideal way to answer this question, it is hard to imagine them being undertaken because most trials to date have compared cementing systems and not alternative types of restorations.

\section{Practice point}

- IPS-Empress inlay, onlays and anterior crowns show good survival in the short term. Comparative studies are needed to determine if they are the best restoration for a particular tooth.

\section{Barbara Chadwick}

Senior Lecturer Paediatric Dentistry, Paediatric Dentistry Unit, Dental School, University of Wales College of Medicine, Cardiff, Wales, UK

1. Martin N, Jednakiewicz NM. Clinical performance of CEREC ceramic inlays: a systematic review. Dent Mater 1999; 15:54-61.

2. Hayashi M, Yeung CA. Ceramic inlays for restoring posterior teeth (Cochrane Review). In the Cochrane Library. Chichester: John Wiley; 2004; issue 2.

3. Chadwick BL, Dummer PMH, Dunstan F et al. The Longevity of Dental Restorations. A Systematic Review. York: University of York NHS Centre for Reviews and Dissemination 2001; 19:27-32.

Evidence-Based Dentistry (2004) 5, 73.

doi:10.1038/sj.ebd.6400265 